$16^{\text {th }}$ International Congress of Metrology, 09002 (2013)

DOI: $10.1051 /$ metrology/201309002

(c) Owned by the authors, published by EDP Sciences, 2013

\title{
Cost-benefit analysis of renewable energy systems under uncertainties
}

\author{
Emmanouil Mathioulakis ${ }^{\mathrm{a}}$, George Panaras and Vassilis Belessiotis \\ National Center for Scientific Research «DEMOKRITOS» \\ 15310 Agia Paraskevi Attikis, Greece
}

\begin{abstract}
Despite the continuously increasing tension in the field of energy supply, suspicion still remains as regards the renewable energy sources (RES) applications, especially on the level of the actual installation cost and its comparison to the expected benefits. Within this framework, the well-known Cost-Benefit Analysis (CBA) technique is widely used. The problem with the usual, conventional way for the implementation of CBA lies in the fact that the values of the cost - benefit parameters are not known precisely, thus undermining the reliability of the results. More specifically, in the case of RES investments, these uncertainties are related either to the monetary values themselves (e.g. buying cost, rate of interest, maintenance cost, future cost of energy, etc.), or to parameters which affect the monetary quantities (e.g. anticipated life time of the product, malfunctions frequency, expected saving of conventional energy, variability of meteorological conditions, uncertainties related to the performance testing results of the systems etc). In the proposed work, a modified version of the CBA based on the Monte-Carlo simulation is discussed. The examined approach is compatible with the current perceptions regarding the treatment of information under uncertainty conditions, through the use of conventional metrological tools.
\end{abstract}

\section{Introduction}

The increasing intensity of the energy supply problems, especially considering fossil fuels availability and the impacts of the climatic change, has contributed to the positive consideration of the renewable energy applications by most of the social partners. This positive attitude towards renewable energy sources (RES) though, is not automatically translated into practical applications, as demonstrated by the increasing but still limited contribution of RES to the energy balance, especially when compared with the exploitable RES potential. The basic reason for this deviation between the desired and the achieved target is related with the actual or supposed cost of RES installations, if compared with the expected benefits.

The effectiveness of the investment in RES systems can reasonably be considered a critical parameter for the individual investor, who desires a profitable, on financial terms, investment, for himself. It also constitutes a basic selection criterion for the institutions or the state authorities, which are called, within the implementation of their energy policy, upon the evaluation of the possible interventions, taking into account the financial cost benefit data, as well as the indirect consequences of these policies in such sectors as health, environment protection or the creation of new employment positions.

\footnotetext{
${ }^{\mathrm{a}}$ Corresponding author: math@ipta.demokritos.gr
}

Within this context, the widely known Cost-Benefit Analysis (CBA) technique is used, which constitutes a classical, and at the same time irreplaceable, tool for the assessment of the reciprocity of any activity involving incoming and outgoing cash flows over a specific time period. The CBA can also be used as a tool for the optimization of the final investment, to the degree that it allows the evaluation of alternative scenarios which differ from one another in terms of the examined technical or administrative preferences.

The CBA aims at an overall assessment of the investment, taking into consideration all the cost and benefit parameters. The result of the analysis can be either the estimation of the expected financial impact of a specific investment, or the comparison between different investment scenarios with regard to the expected financial benefits. Within the framework of the CBA, all the cost and benefit parameters have to be expressed as monetary values, taking into account their escalation over time. The monetary value attributed over the involved quantities represents either commercial values, or it is the resultant of personal preferences combined through the use of specialized techniques, e.g. the Contingent Valuation Method (CVM).

Even though the elaboration of CBA studies can be considered a routine activity, the study of some particular aspects of its application in the field of RES is presented in the relevant literature. For example, the related works 
to the techno-economic evaluation of small hydro power or even the cost benefit analysis of a photovoltaic-energy storage electrification solution for remote islands $([1,2])$ can be referred to. Gocht et al. ([3]) propose an investigation of the technical feasibility, as well as a cost benefit analysis of a brackish water small-scale desalination plant in a rural area, leading to the best socio-economic performance for a reverse osmosis directly coupled with a photovoltaic-system. Fraidenraich et al., ([4]), propose an estimation of a cost-benefit figure, given by the relationship between collectible energy and reflector surface, based on analytic solutions for the geometric and optical properties of stationary compound parabolic concentrators with fully illuminated inverted V receiver. Snyder and Kaiser ([5]) discuss the costs and benefits of offshore wind relative to onshore wind power and conventional electricity production. Finally, particular reference to the work of Diakoulaki et al. ([6]) should take place. The authors present a paper discussing a cost benefit analysis for evaluating solar domestic hot water systems (SDHWS) in comparison with competitive conventional technologies in Greece. The major external benefits included in the proposed analysis are energy saving, avoidance of environmental impact and job creation.

One of the most important problems of the CBA is the fact that the quantities entering the analysis are often characterized by significant uncertainties, especially as regards the financial quantities ([7]). For the particular cases, where the scope is the elaboration and assessment of policies and incentives for supporting RES, the uncertainties related with the monetary values can be quite significant, thus undermining the credibility of the analysis $([6,8])$. For the case of RES, the uncertainties are related either with the purely monetary values (e.g. buying cost, interest, maintenance cost, future cost of energy, etc.), or with technical parameters which influence the monetary quantities (e.g. useful life cycle, failure frequency, expected saving of conventional energy) indirectly. For the particular case of solar thermal systems, which is examined in more detail later on, the expected benefit is characterized by uncertainties related to the metrological equipment through testing, the imperfect knowledge of the meteorological conditions which might take place through the actual operation of the system, the assumption of a typical load profile different from the one to be implemented by the potential user, or even more related to the future value of the conventional fuel which would be substituted by the solar system.

A usual approach for the treatment of uncertainties comprises the elaboration of sensitivity analysis, aiming at the investigation of the variation of the quantitative conclusions of the analysis through the whole variation range of every uncertain parameter ([9]). However, while a sensitivity analysis tries to identify which source of uncertainty weights more on the study's conclusions, an uncertainty analysis has to produce an estimation of the overall uncertainty in the conclusions of the study, based on a realistic picture of the uncertainty characterizing the different sources of variation in the input of the analysis.
In the proposed work a modified version of the CBA is discussed, which is based on the Monte-Carlo simulation and it is compatible with the current perceptions regarding the treatment of information under uncertainty conditions. The proposed approach takes advantage of the available state-of-knowledge regarding the cost and benefit analysis parameters, as this is encoded through the use of appropriate probability distributions. The classic CBA is used as a model for the elaboration of the available information; together with the Monte-Carlo simulation, and through the implementation of the propagation of distribution technique, a stochastic image for the quantitative results of the CBA is built.

Initially, a brief reference to the methodological framework of the CBA, as well as to the basic principles for the treatment of the available information under uncertain conditions through the use of probability distributions is performed. Later on, the propagation of distributions on the basis of the Monte-Carlo simulation is discussed, as well as the potential to proceed to a realistic shaping of the result through the synthesis of individual uncertain information. Finally, a typical example for the implementation of the proposed methodology in the case of solar thermal energy applications in the domestic sector is provided.

\section{CBA analysis}

In brief, the stages through which the CBA analysis of a specific project comes through are the following ([6, 9, 10]):

I. Identification, with the maximum possible accuracy, of technical and functional parameters of the installation to be implemented through the project.

II. Determination, with the maximum possible accuracy, of the cost and benefit components of the project, on the basis of all information which can be attained, as market data, experience by similar installations, calculations, etc.

III. Expression of all cost and benefit components on a common basis in terms of their Present Value (PV), taking into consideration their escalation in time through the total life cycle of the investment.

IV. Assessment of the indices for the economical effectiveness of the investment.

This assessment can be performed on the basis of various indices; the more usual ones refer to the Net Present Value (NPV), the Internal Rate of Return (IRR) and the Benefit Cost Ratio $(B C R)$.

To compute all relevant future costs and benefits in present-value terms, a discount rate is chosen, most commonly in connection to an interest rate taken from financial markets. Suppose a project involves benefits (revenues) and costs over a time span from the present (time 0 ) to $n$ years from now. Let $B_{t}$ and $C_{t}$ refer, respectively, to the benefit and cost $t$ years from now, and let $r$ represent the annual rate of return on a standard investment. Then, the present value of the net benefit $(P V N B)$ would be given by: 


$$
P V N B=\sum_{t=0}^{n} \frac{B_{t}-C_{t}}{(1+r)^{t}}
$$

Despite its simplicity the NPV index presents the drawback of adopting a specific value for the annual rate of return $r$, while it cannot be used for the rating of investments independent from one another, as it is an absolute and not a relative index. The same disadvantages can also be attributed to the $B C R$.

The internal rate of return (IRR) is the critical interest rate (where $N P V=0$ ) and it should not be lower than the interest rate used, in order for an investment to be economically sustainable. IRR can be calculated from the following equation:

$$
\sum_{t=0}^{n} \frac{B_{t}-C_{t}}{(1+I R R)^{t}}=0
$$

This index is often proven to be the most suitable for the rating of different projects as it does not present any dependence on the selection of the rate of return $r$; the distance by the chance cost of the capital comprises the evaluation criterion. For this reason, this work concentrates on the assessment of the reciprocity of the candidate projects under uncertainty conditions, even though a similar analysis can take place on the basis of other indices as well.

Equation 2 can be considered to constitute a measurement model under a wide perspective, to the degree that it combines for every investment some initial information (time period $n$ of the useful investment life, benefits $B_{t}$ and costs $C_{t}$ ) for calculating the value $r$ of IRR (figure 1).

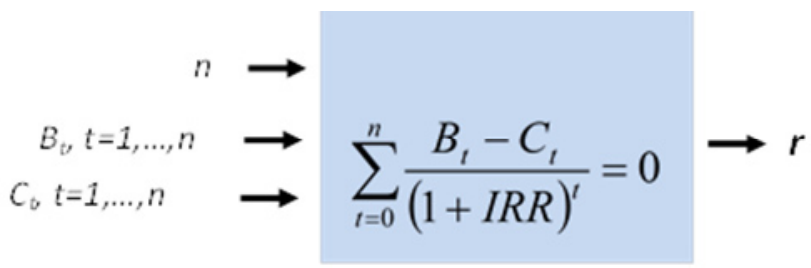

Figure 1. Measurement model of the IRR

As it has been mentioned, the input information, as well as the result of the calculation are characterized by uncertainties which propagate through the IRR calculation model. Moreover, to the degree that this calculation is performed iteratively, the analytical calculation of the partial derivatives of $r$ with regard to the input quantities is not possible. This inability does not allow the implementation of the classical error propagation approach, a fact which constitutes the propagation of distributions via the Monte-Carlo simulation an almost unavoidable alternative preference.

\section{The Monte-Carlo approach}

The weaknesses of the conventional error-propagation method for uncertainty estimation, combined with the rapid increase of the computational capacity available to the laboratories, have favored the dissemination of an alternative approach, referred to as the Monte Carlo technique, which has been the subject of the first addendum to GUM $([11,12,13])$. The basic idea of this technique concerns the propagation of distribution rather than the propagation of the uncertainties. In the case of a measurement model $y=f\left(x_{1}, \ldots, x_{M}\right)$ which calculates the value $\mathrm{y}$ of the measurand $\mathrm{Y}$ as a function of the experimental values $x_{1}, \ldots, x_{M}$ of the $\mathrm{M}$ physical quantities $X_{1}, \ldots, X_{M}$, the implementation of the method can be summarized as follows:

- For each measurement point, the information about a given value $\mathrm{xi}$ of the input quantity $\mathrm{Xi}$ is encoded by a specified Probability Distribution Function (PDF) $g_{x_{1}}$. This PDF can be experimentally inferred from direct repeated measurements of the input quantity or assigned to the primary input estimate on the base of the Principle of Maximum Entropy ([14]).

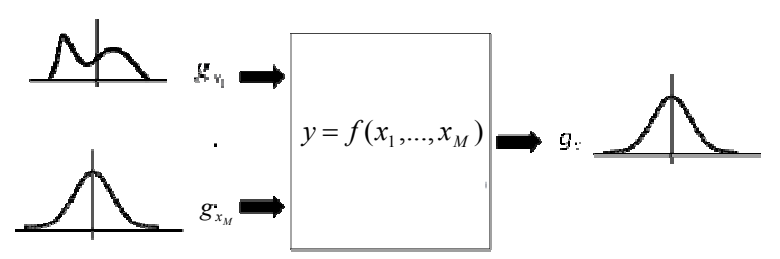

Figure 2. Schematic representation of the Monte-Carlo approach

- A suitable algorithm is used to generate, for each primary source value $x_{i}$, a high number sequence of $\mathrm{N}$ values $x_{i, j}, j=1, \ldots, N$, the statistical properties of which approximate those of the respective PDF $g_{x_{1}}$

- The measurement model $y=f\left(x_{1}, \ldots, x_{M}\right)$ is used $\mathrm{N}$ times, one time for each of the $\mathrm{N}$ combinations $x_{1}, j, \ldots, x M, j, j=1, \ldots, N$. Thus, $\mathrm{N}$ values $y_{j}, j=1, \ldots, N$ of the result are produced (Fig. 2), allowing the formulation of a discrete representation $g_{y}$ of the PDF for the measurand $\mathrm{Y}$.

- The distribution $g_{y}$ allows the calculation of the average $\bar{y}$ and standard deviation $u_{y}$, considered the best possible estimates of the value of $Y$ and of the associated standard uncertainty:

$$
\begin{gathered}
\bar{y}=\frac{1}{N} \sum_{i=1}^{N} y_{i} \\
u_{y}=\sqrt{\frac{1}{N-1} \sum_{i=1}^{N}\left(y_{i}-\tilde{y}\right)^{2}}
\end{gathered}
$$




\section{Application to the case of solar domestic hot water systems (SDHWS)}

The proper quantitative assessment of the input uncertainties, that is the use of the proper probability distributions for each one of them, is considered critical for the extraction of reliable conclusions. This quantification can be based on measurements, estimations, considerations by experts, simulation results, physical limitations, market surveys or pre-existing experience.

The cost related to the initial purchase and maintenance can be easily extracted from the market, and proves to be more or less uncertain depending on the examined case. If the question considers a specific solar thermal system, the uncertainty is limited to the maintenance cost and the duration for the exploitation of the investment. In the case though of a category of products, as for example all the systems of a specific size available on the market, the information concerning the purchase cost is uncertain and can be represented by an orthogonal distribution of a range equal to the complete range of the market cost.

The information which can be characterized as even more uncertain is the one related to the expected benefits, and more specifically the ones which are of direct interest for the user; in this sense, the externalities are not included ([6]). In the case of Greece, these benefits concern the cost of saved electrical energy, given that in Greece the domestic hot water is almost exclusively heated through the use of electrical energy. For other uses, as for example that of space heating, the specific energy mixture used has to be introduced in the analysis. Moreover, through the calculation of the expected benefits, the potential existence of tax or other type of incentives has to be taken into account; for the needs of the specific application, these incentives are considered to be zero.

In general, the decision for considering a specific parameter as known or uncertain can be based on various criteria. Such criteria concern the scope of the project, the available information and the resources that can be spent to conduct the analysis. For most cases it is preferable to consider as uncertain most of the influential parameters, while constant values are considered (point estimates) for the rest.

Following on, a typical application with particular interest for countries like Greece, where almost the total installed collector area of 4 million $\mathrm{m}^{2}$ concerns solar domestic hot water systems (SDHWS), is presented. The objective is the investigation of the reciprocity of an investment for a typical SDHWS under uncertain conditions, and more specifically the assessment of the $I R R$ index.

A specific group of products is examined; these products present satisfactory performance and come from recognized manufacturers. They belong to the widely disseminated category of typical SDHWS with a solar storage volume in the order of 2001 and almost $4 \mathrm{~m}^{2}$ of collector gross surface. The investigation is performed for typical use of complete exploitation of the collected energy, as this is described in the relevant standards for the area of Athens ([15]). The analysis considers that the saved conventional energy comes from the domestic electrical energy network. It should be noted that the methodology adopted for the investigation would be primarily the same for other categories of products or for an individual product.

For the quantification of input uncertainties for the specific application, the collection and elaboration of information regarding the initial buying and maintenance cost of the SDHWS, as well as of information regarding the expected benefit was performed. More specifically, the following working hypotheses were adopted:

- The initial cost of the examined group of products lies from $1100 €$ up to $1500 €$. Due to the limited number of commercially available products of the examined group, the relevant information is expressed with the help of a rectangular pdf, presenting a mean value of $1300 €$ and a range of $\pm 200 €$.

- For the life duration of the investment, historical data by manufacturers have been used. According to these data, the expected life time of the SDHWS can be considered that as following the normal distribution with an expectation of 15 years and a standard deviation of 2.5 years.

- For the maintenance cost, the rectangular distribution is also assumed; the extreme values are $15 €$ and $25 € /$ year.

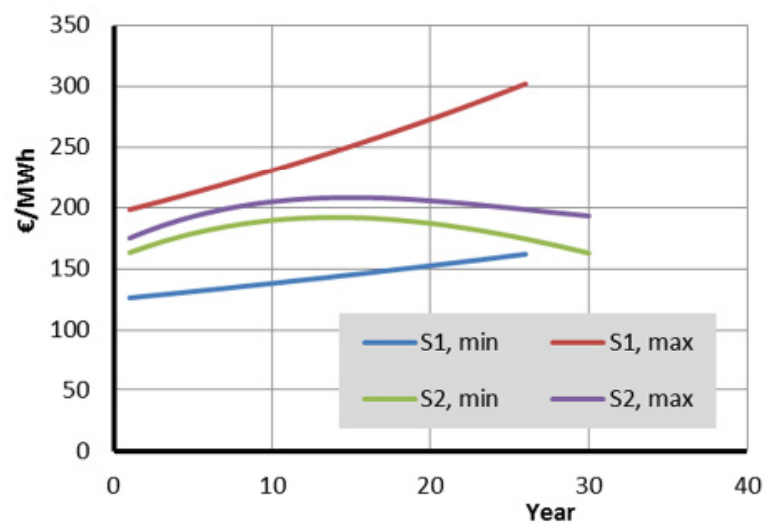

Figure 3. Progress of the electrical energy price for domestic use

For the assessment of the expected benefit, the expected performance of the system and the cost of the replaced conventional energy are taken into account. For the former, the relevant information comes from the testing results, taking into account the standard uncertainty which characterizes this result ([16]). For the cost of the conventional energy, within the framework of the present work two scenarios have been examined. The scenario S1 is based on the existing gradual convergence of the electrical energy prices on a European level, taking into account the current prices and the differences between the various categories of pricing. 
This scenario assumes a reasonable error margin, and concludes on values which are expected to lie between the values of the two curves S1, min and S1, max of figure 3 ([17]); a probability level of $95 \%$ is assumed for the provided estimations. This estimation is compatible with a second, more conservative scenario, referred to as S2, adjusted on the characteristics and data of the Greek sector on a higher degree; moreover, this scenario often appears in the relevant literature ([18]). Thus, for each year, a normal pdf is assumed with an expectation equal to the mean value and a standard deviation equal to the half difference between the maximum and mean value.

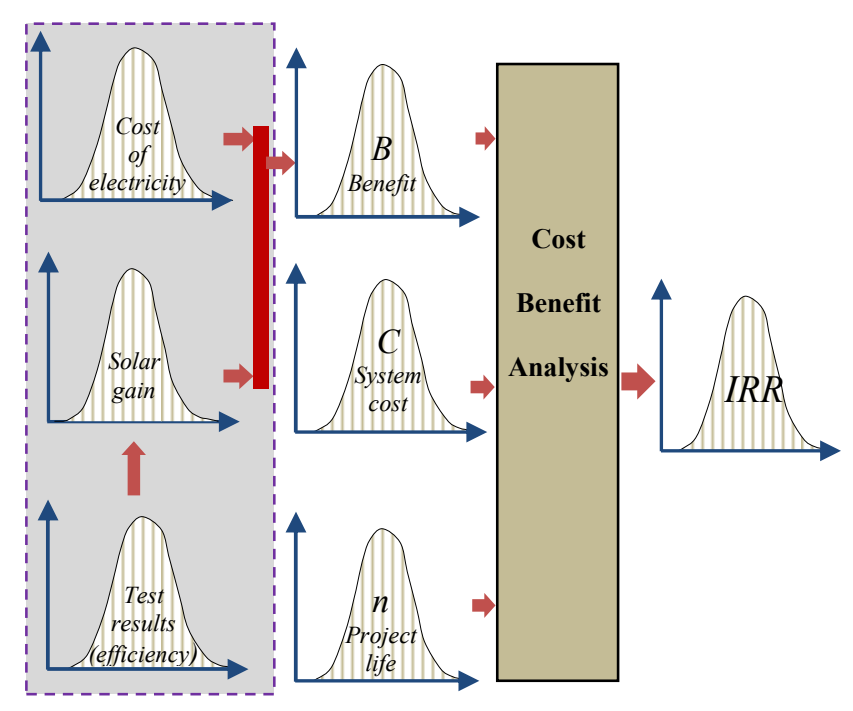

Figure 4. Propagation of distributions

The flow of information from the primary data to the final result is depicted in figure 4. Actually, the complete calculation procedure is treated as a measurement; the uncertain initial information, expressed through appropriate probability distributions, propagates to the final result. The implementation of the proposed methodology to the data of the specific case studied in this work, led to the results presented in figure 5 .

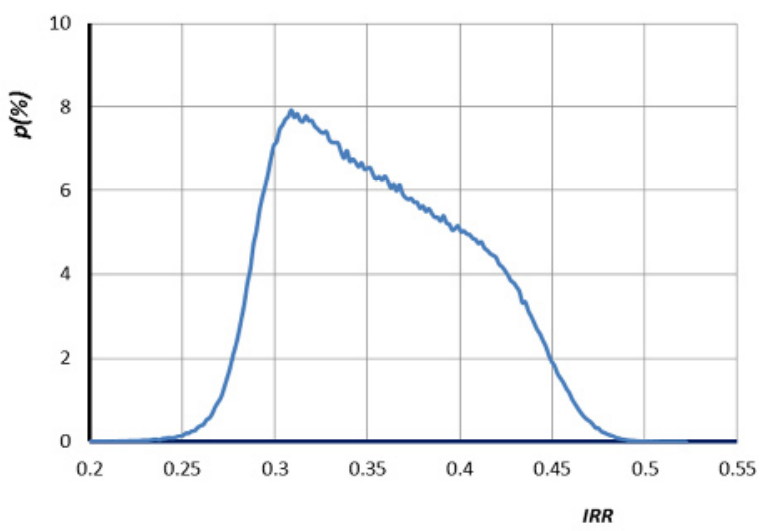

Figure 5. Probability distribution for $I R R$

The determination of the $I R R$ probability density function may lead to useful results, such as the expectation $\overline{I R R}=0.35$ or the $I R R$ standard deviation
$S_{i r r}=0.05$. Moreover, the probability for the profitability of the investment to exceed specific criteria can be deduced by proper integration of the specific pdf; in fig.6, for example, the probability $p_{r}$ for the IRR of the investment being greater than a given value of $r$ is presented.

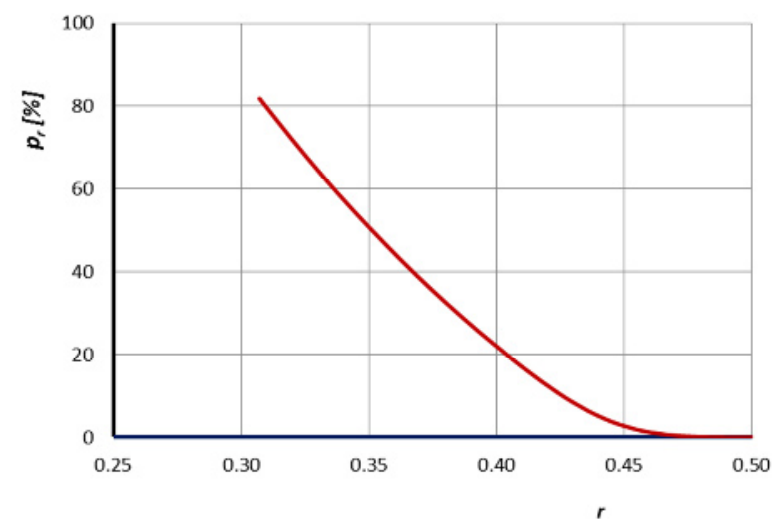

Figure 6. Probability $p_{r}$ for the $I R R$ being greater than a given value of $r$

It can be noted that the implementation of a similar methodology may be used for the estimation of the value of other indices quantifying the profitability of the investment, such as the $N P V$ and the $B C R$.

\section{Conclusions}

The assessment of the indices describing the effectiveness on an economical level of an investment concerning the exploitation of renewable energy sources, has to face the difficulties raised by the existence of significant uncertainties on the values of the parameters affecting this estimation. The quantitative estimation of the effectiveness indices under uncertainty conditions can thus be treated as an uncertainty propagation problem, through the use of the ordinary tools of metrology.

On the specific work, emphasis is given to the formulation of a methodology for the acquisition of the stochastic information related to a specific index for the economical evaluation of an investment on a solar domestic hot water system; the methodology is based on the propagation of distribution technique. This technique takes advantage of the Monte-Carlo simulation, widely used on metrological applications, as this is considered to be the most appropriate one for cases where the analytical calculation of the sensitivity coefficients of the measurement model is not possible.

The implementation of the proposed methodology enables the assessment of the probability for the Internal Rate of Return presenting specific values, thus allowing the candidate user to extract realistic conclusions as regards the effectiveness of the investment.

\section{References}

1. Kaldellis J. K., Vlachou D. S., Korbakis G., Energy Policy, 33, 15 (2005). 
2. Kaldellis J.K., Zafirakis D., Kaldelli E.L., Kavadia K., Renewable Energy, 34, 5 (2009).

3. Gocht W., Sommerfeld A., Rautenbach R., Melin Th., Eilers L., Neskakis A., Herold D., Horstmann V., Kabariti M., Muhaidat A., Renewable Energy, 14, 1-4 (1998).

4. Fraidenraich N., Tiba C, Brandão B., Vilela O., Solar Energy, 82, 2 (2008).

5. Snyder B., Kaiser M. J., Renewable Energy, 34, 6 (2009).

6. Diakoulaki D., Zervos A., Sarafidis J., Mirasgedis S., Energy Conversion and Management, 42, 14 (2001).

7. Boardman, A.E., Greenberg, D.H., Vining, A.R. and Weimer, D.L., Cost-Benefit Analysis: Concepts and Practice (Prentice Hall, New Jersey, 1996).

8. Goulder L. H., Stavins R. N., Nature, 419, 17 (2002).

9. Kopp R., Krupnick A., Toman M., Discussion Paper 97-19, Resources for the Future, (1997).

10. Brent R. J., Applied Cost-Benefit Analysis (Edward Elgar Publishing, 2006).

11. BIPM, IEC, IFCC, ILAC, ISO, IUPAC, IUPAP, OIML, 2008. Evaluation of Measurement Data Supplement 1 to the 'Guide to the Expression of Uncertainty in Measurement - Propagation of Distributions Using a Monte Carlo Method (BIPM, Paris, 2008).
12. Wubbeler, G., Krystek, M., Elster, C., Measurement Science and Technology, 19, 8 (2008).

13. Cox, M.G., Siebert, B.R.L., Metrologia, 43 (2006).

14. Lira, I., Elster, C., Wöger, W., Cox, M.G., Advanced Mathematical \& Computational Tools in Metrology VIII, in Series on Advances in Mathematics for Applied Sciences, 78, (2009).

15. CEN European Standard EN12976-2:2006, Thermal Solar Systems and Components - Factory Made Systems - Part 2: Test Methods (CEN, Brussels, 2006).

16. Mathioulakis E, Panaras G., Belessiotis V., Solar Energy, 86, 11 (2012).

17. European Commission, EU Energy Roadmap 2050, (European Commission, Directorate-General for Energy, 2011).

18. Kapros P, Long-term energy perspectives for Greece - PRIMES model (in Greek), (IOBE ed., Athens, 2011). 